\title{
Emergency Evacuation Simulator (EES) - a Tool for Planning Community Evacuations in Australia (Demonstration)
}

\author{
Dhirendra Singh and Lin Padgham \\ RMIT University, Australia \\ dhirendra.singh@rmit.edu.au, lin.padgham@rmit.edu.au
}

\begin{abstract}
This work addresses the problem of encoding cognitive agents that are capable of complex reasoning beyond simple rules, within agent-based models (ABM). This is particularly important for social simulation where agents represent people. We provide a general solution to this problem through infrastructure that allows the integration of stateof-the-art Belief-Desire-Intention (BDI) and ABM systems. In this paper, we demonstrate how this infrastructure is being used to help emergency services in Australia plan for community evacuations.
\end{abstract}

\section{Introduction}

For the past few years we have been working with emergency services in Australia on a simulation tool-Emergency Evacuation Simulator (EES)-that can be used to build community evacuation scenarios to aid understanding and planning. Figure 1 shows an example view of an evacuation produced by our tool. The large red patch shows a progressing fire front to the northwest of town. Evacuating vehicles are colour-coded to indicate speeds-from green (free flowing) through to red (slow) giving instant visual feedback on traffic conditions. Together with analysis of egress times the tool can provide valuable understanding of likely and worst case scenarios.

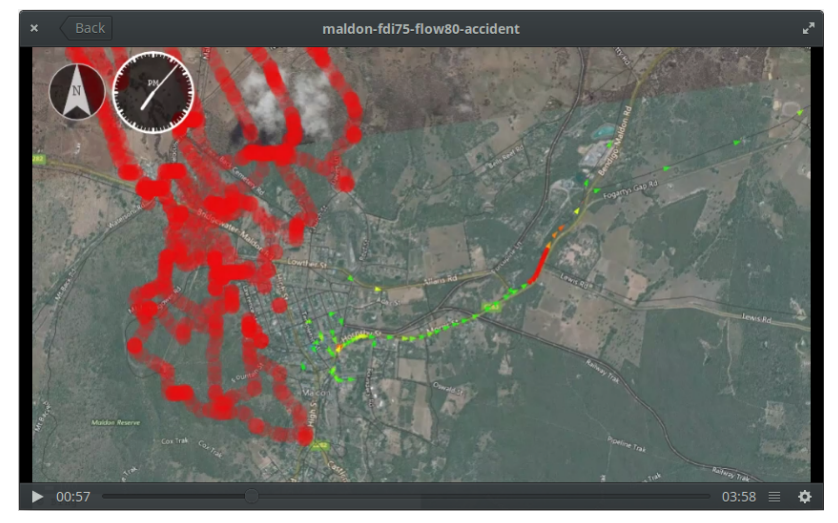

Figure 1: Example evacuation produced by EES.

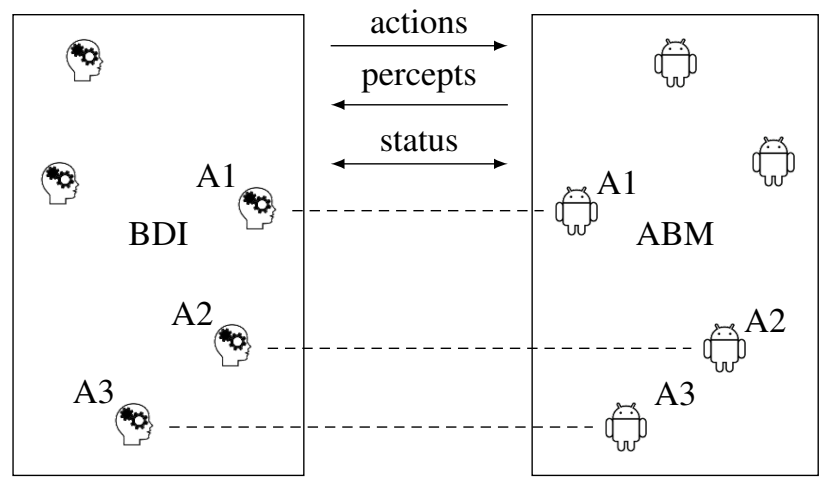

Figure 2: Conceptual BDI-ABM integration architecture

The development work-flow has typically involved an initial meeting with agencies to develop the scenario details, coding the scenario, and then meeting again to discuss results and refine further in an iterative way. However, naturally this process was dependent on availability of resources and personnel, and a single iteration could take weeks. More recently we have built an industry grade web-based interface for the simulator, that allows working groups to visually construct an evacuation scenario and obtain simulation results very quickly-within minutes. This has greatly aided usability.

\section{BDI-ABM Integration Framework}

Technically, EES uses our general-purpose BDI-ABM infrastructure [Singh et al., 2016] that allows agent-based simulations to be built where conceptually the "brains" of some agents exist in a Belief-Desire-Intention (BDI) reasoning system, e.g., JACK [Busetta et al., 1998], while their "bodies" live in the agent-based model (ABM), e.g., MATSim [Horni et al., 2016] traffic simulator (Figure 2). We use BDI [Rao and Georgeff, 1991] for cognitive modelling as it provides an established and robust computational model, captures richness of behaviour in a compact visually-presentable hierarchical representation of goals and plans, and allows behaviours to be readily understood and (informally) validated by domain experts. The setup still conforms to the standard paradigm of situated agents that continuously perceive changes in the environment, and perform actions that cause further changes to it. 


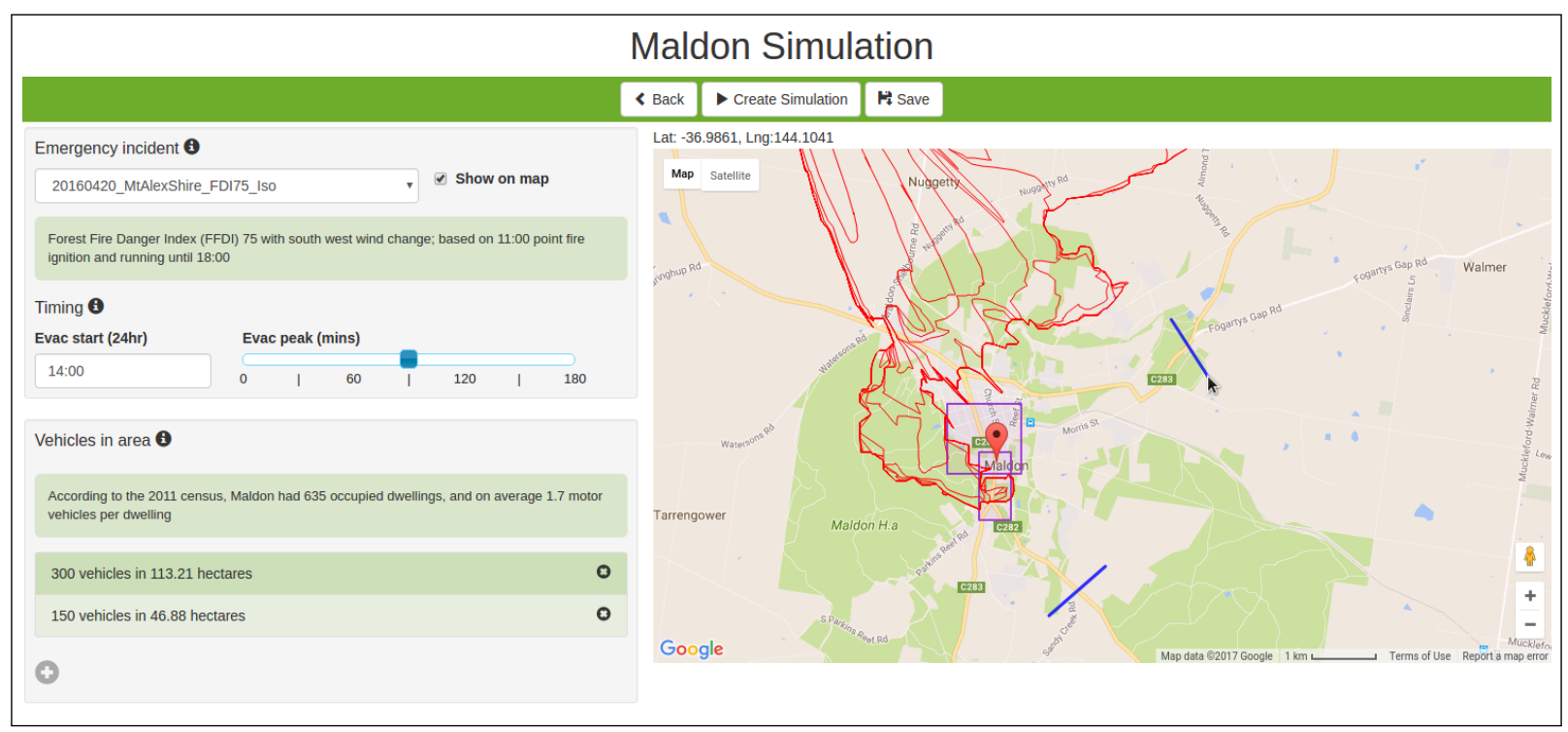

Figure 3: The EES web-based user interface for specifying an evacuation scenario.

Architecturally, the framework consists of three distinct layers. A generic layer realises the conceptual model shown in Figure 2 and provides the functionality for packaging information about actions and percepts passed between the ABM and BDI systems at each time-step. The system layer provides the code necessary for linking a particular BDI system or ABM system into the generic layer. Here, the BDI System Integration has three main functions, to (a) provide a transparent programming model for durative actions, (b) collect actions from, and distribute percepts to, individual agents (c) detect the end of a BDI reasoning cycle. The ABM System Integration on the other hand serves to (a) update actions status', (b) perform an ABM time step, and (c) distribute actions to, and collect percepts from, individual agents. Finally, the application layer provides the domain specific code to form an executable program.

So far we have developed BDI integrations for JACK [Busetta et al., 1998], Jadex [Braubach et al., 2005], and GORITE [Jarvis et al., 2013], and ABM integrations for Repast [North et al., 2007], GAMS [GAMS Development Corporation, 2015], MATSim [Padgham and Singh, 2016], and a Python-based simulator [Geard et al., 2013]. As well, we have presented example uses of the BDI-ABM framework for community evacuations [Padgham et al., 2011; Padgham et al., 2014; Singh and Padgham, 2015], parental decision-making around child vaccinations [Geard et al., 2013], and reverse-auction incentive schemes for conservation targets [Singh et al., 2016].

\section{Simulating Community Evacuation Scenarios}

In this demo, users will get hands-on experience in the use of the web-based EES tool for building community evacuation scenarios. We will provide a short written description of the emergency scenario. Users will then be asked to visually construct the scenario within EES. Specifically, they will:

- select a bushfire model within the tool and understand the likely impact of the progressing fire front on a town;

- choose sensible settings for the timing of the evacuation alert (as well as the peak in departure times of vehicles) with respect to the fire, based on their estimate of how long it would take to establish the severity of the fire and community members to respond;

- specify the number of vehicles, their locations, destinations they would evacuate to, and safe lines that should be monitored in the simulation for egress times; and

- run the simulation and interpret results.

The user sessions are expected to take 10-15 minutes each, and also offer opportunities for observing attendees to participate along with the user attempting the task. At the end, we will ask users for quick feedback on various aspects of the tool, including usability, and value of provided results and analysis from various perspectives. We hope to extract valuable suggestions and insights from an international audience, given that so far the tool has only been trialled in Australia.

Finally, we will have at hand information about the underlying technologies including the BDI-ABM framework, for attendees who may be interested in applying cognitive reasoning to simulations in their own work.

\section{Acknowledgments}

We thank Telematics Trust and Mount Alexander Shire Council for funding parts of this work, and Mount Alexander Shire Fire Management Committee for helping specify, test, and evaluate the web-based EES version. 


\section{References}

[Braubach et al., 2005] Lars Braubach, Alexander Pokahr, and Winfried Lamersdorf. Jadex: A BDI agent system combining middleware and reasoning. In Software Agent-Based Applications, Platforms and Development Kits, pages 143-168, 2005.

[Busetta et al., 1998] Paolo Busetta, Ralph Rönnquist, Andrew Hodgson, and Andrew Lucas. JACK intelligent agents - components for intelligent agents in Java. Technical report, AOS Pty. Ltd, Melbourne, Australia, 1998.

[GAMS Development Corporation, 2015] GAMS Development Corporation. General algebraic modeling system (gams) website. http: / / www . gams . com/, 2015. Accessed: Feb 19, 2016.

[Geard et al., 2013] Nic Geard, Dhirendra Singh, Jodie McVernon, and Lin Padgham. A model of parental decision making and behaviour about childhood vaccination. In Epidemics 4: Fourth International Conference on Infectious Disease Dynamics, pages 19-22, Amsterdam, The Netherlands, November 2013.

[Horni et al., 2016] A. Horni, K. Nagel, and K.W. Axhausen, editors. The Multi-Agent Transport Simulation MATSim. Ubiquity Press, London, 2016.

[Jarvis et al., 2013] Dennis Jarvis, Jacqueline Jarvis, Ralph Rnnquist, and Lakhmi C. Jain. Multiagent Systems and Applications - Volume 2: Development Using the GORITE BDI Framework, volume 46 of Intelligent Systems Reference Library. Springer, 2013.

[North et al., 2007] M. J. North, T. R. Howe, N. T. Collier, and J. R. Vos. Advancing Social Simulation: The First World Congress, chapter A Declarative Model Assembly Infrastructure for Verification and Validation, pages 129140. Springer Japan, Tokyo, Japan, 2007.

[Padgham and Singh, 2016] Lin Padgham and Dhirendra Singh. The Multi-Agent Transport Simulation MATSim, chapter Making MATSim agents smarter with the BeliefDesire-Intention framework. Ubiquity Press, London, 2016.

[Padgham et al., 2011] Lin Padgham, David Scerri, Gaya Buddhinath Jayatilleke, and Sarah L. Hickmott. Integrating BDI reasoning into agent based modeling and simulation. In Winter Simulation Conference (WSC), pages 345-356, December 2011.

[Padgham et al., 2014] Lin Padgham, Kai Nagel, Dhirendra Singh, and Qingyu Chen. Integrating bdi agents into a matsim simulation. Frontiers in Artificial Intelligence and Applications, 263(ECAI 2014):681-686, 2014.

[Rao and Georgeff, 1991] A.S. Rao and M.P. Georgeff. Modeling rational agents within a BDI-architecture. In Principles of Knowledge Representation and Reasoning (KR), pages 473-484, Cambridge, MA, USA, 1991.

[Singh and Padgham, 2015] Dhirendra Singh and Lin Padgham. Community evacuation planning for bushfires using agent-based simulation (demonstration). In
Autonomous Agents and Multi-Agent Systems (AAMAS), pages 1903-1904, Istanbul, Turkey, May 2015.

[Singh et al., 2016] Dhirendra Singh, Lin Padgham, and Brian Logan. Integrating BDI agents with agent-based simulation platforms. Autonomous Agents and MultiAgent Systems, 30(6):1050-1071, 2016. 